\title{
Inhibition of fatty acid synthase suppresses osteosarcoma cell invasion and migration via downregulation of the PI3K/Akt signaling pathway in vitro
}

\author{
ZHI LI LIU ${ }^{1 *}$, JIAN HUA MAO ${ }^{1 *}$, AI FEN PENG ${ }^{2}$, QING SHUI YIN ${ }^{3}$, \\ YANG ZHOU ${ }^{1}$, XIN HUA LONG ${ }^{1}$ and SHAN HU HUANG ${ }^{1}$ \\ ${ }^{1}$ Department of Orthopedics, First Affiliated Hospital of Nanchang University; \\ ${ }^{2}$ Jiangxi University of Traditional Chinese Medicine, Nanchang 330006; ${ }^{3}$ Hospital of Orthopedic Surgery, \\ Guangzhou General Hospital of Guangzhou Military Region, Guangzhou 510080, P.R. China
}

Received June 22, 2012; Accepted October 24, 2012

DOI: $10.3892 / \mathrm{mmr} .2012 .1220$

\begin{abstract}
In the present study, the effect of fatty acid synthase (FASN) inhibition on cell invasion and migration in vitro was investigated. A recombinant plasmid containing a microRNA targeting the FASN gene was used to inhibit FASN expression in U2-OS cells. Cell migration and invasion were investigated using wound healing and Transwell invasion assays. We found that cell invasion and migration were suppressed by inhibiting FASN. In addition, the effect of inhibition of FASN on phosphorylation of Akt was investigated by detecting the expression levels of pAkt using western blot analysis. Furthermore, protein expression levels of nuclear factor- $\kappa \mathrm{B}(\mathrm{NF}-\kappa \mathrm{B} ; \mathrm{p} 65)$ and matrix metalloproteinase (MMP)-2 and -9 were also measured by western blot analysis. Results demonstrated that expression levels of pAkt, NF- $\mathrm{B}$ (p65) and MMP-2 and -9 proteins were reduced significantly by inhibiting FASN. Therefore, we confirmed that inhibition of FASN by RNA interference suppresses osteosarcoma cell metastasis via downregulation of the phosphoinositide 3-kinase/Akt/NF- $\kappa \mathrm{B}$ signaling pathway in vitro.
\end{abstract}

\section{Introduction}

Osteosarcoma (OS) is one of the most common primary malignant bone tumors in children and adolescents. In the early 1970 s, introduction of doxorubicin and methotrexate with leucovorin rescue demonstrated promise for the improvement of OS patient survival. The five-year survival rate for

Correspondence to: Professor Shan Hu Huang, Department of Orthopedics, First Affiliated Hospital of Nanchang University, Yong Wai Zheng Street 17, Nanchang, Jiangxi 330006, P.R. China E-mail: hsh869@126.com

*Contributed equally

Key words: fatty acid synthase, metastasis, osteosarcoma, PI3K/Akt patients treated with intensive multidrug chemotherapy and aggressive local control has been reported at 55-80\% (1-3). However, despite the encouraging trend for longer survival, many patients still have a poor prognosis. Previous studies have estimated the five-year survival rate of patients with metastatic diseases to be $<20 \%$ (4-6). Development of lung metastases is the main cause of mortality in patients with OS. Therefore, identification of the molecular mechanisms of metastasis in OS is likely to have a significant impact on management and prognosis of the disease.

Fatty acid metabolic pathways have been previously reported to be associated with carcinogenesis (7). Fatty acid synthase (FASN) is an important enzyme involved in endogenous lipogenesis in mammals and is responsible for catalyzing the synthesis of long-chain fatty acids. FASN has been identified as crucial for sustaining a number of biological features of cancer cells (8). The enzyme is expressed at high levels in a variety of human tumors (8-13), but remains at low levels in normal tissues. Various studies have reported that inhibition of FASN expression may suppress cancer cell proliferation in vitro and in vivo (14-18). In addition, FASN has also been hypothesized to contribute to cancer cell metastasis $(19,20)$. However, it is currently unclear whether this molecule is involved in OS metastasis and the molecular mechanisms associated with FASN and metastasis remain unknown.

Matrix metalloproteinases (MMPs) are involved in degradation of the basement membrane and epimatrix, among which MMP-2 and -9 markedly correlate with tumor invasion and metastasis. MMP-2 and -9 are overexpressed in OS and promote $\mathrm{OS}$ cell migration and invasion by degrading components of the basement membrane and epimatrix. A large number of studies indicate that activation of the nuclear factor- $\kappa \mathrm{B}(\mathrm{NF}-\kappa \mathrm{B})$ gene, an upstream regulator of MMPs, is closely associated with tumor invasion and migration $(21,22)$. In addition, phosphorylation and activation of Akt has been recognized as an important regulatory factor in $\mathrm{NF}-\kappa \mathrm{B}$ signaling. Specifically, activation of Akt has been identified to be essential for degradation of an inhibitor of $\mathrm{NF}-\kappa \mathrm{B}$, inhibitor of $\kappa \mathrm{B}(\mathrm{I} \kappa \mathrm{B})$ and NF- $\kappa \mathrm{B}$ activation mediated by I $\mathrm{KB}$ kinases 
(IKKs) (23). Previous studies have demonstrated that fatty acids synthesized by FASN are incorporated into membrane phospholipids, known modulators of Akt activation $(24,25)$. Wang et al reported that inhibiting FASN decreases phosphorylation of Akt in ovarian cancer cells $(26,27)$. Therefore, we hypothesize that inhibition of FASN may suppress osteosarcoma cell invasion and migration by downregulation of the phosphoinositide 3-kinase (PI3K)/Akt/NF- $\kappa \mathrm{B}$ signaling pathway.

In the present study, the effect of FASN inhibition on OS cell invasion and migration was investigated in vitro. In addition, the effect of inhibition of FASN on the PI3K/Akt/NF- $\mathrm{BB}$ signaling pathway was investigated. Results indicate that inhibition of FASN may inhibit OS cell invasion and migration via downregulation of the $\mathrm{PI} 3 \mathrm{~K} / \mathrm{Akt} / \mathrm{NF}-\kappa \mathrm{B}$ pathway in vitro.

\section{Materials and methods}

Contruction of the recombinant plasmid containing miRNA targeting the FASN gene. The human cDNA sequence encoding FASN protein (NM_004104.4) was obtained from GenBank and miRNA and control single-strain DNA oligos were designed and synthesized using the following primer sequences: forward 5'-TGCTGAACTCCTGCAAGTTCT CCGACGTTTTGGCCACTGACTGACGTCGGAGATTGC AGGAGTT-3' and reverse 5'-CCTGAACTCCTGCAATCT CCGACGTCAGTCAGTGGCCAAAACGTCGGAGAACTT GCAGGAGTTC-3'. Products were cloned into the express vector pcDNA6.2-GW/EmGFP-miR using the BLOCK-iT ${ }^{\mathrm{TM}}$ Pol II miR RNAi Expression Vector kit with EmGFP (K4936-00; Invitrogen Life Technologies, Carlsbad, CA, USA). The DNA sequence of the plasmid was confirmed using the PureLink HiPure Plasmid DNA kit (K2100-03; Invitrogen Life Technologies).

Cell culture and transfection. Human OS cell line, U2-OS, (Shanghai Cell Bank, Chinese Academy of Sciences, Shanghai, China) was cultured in DMEM with $10 \%$ fetal bovine serum (FBS) and incubated at $37^{\circ} \mathrm{C}$ in $5 \% \mathrm{CO}_{2}$. U2-OS cells were seeded in 6-well plates at $30 \%$ confluence on the day prior to transfection. Transfection with recombinant plasmid targeting the FASN gene or negative plasmid was performed using Lipofectamine 2000 reagent. Transfection complexes were prepared according to the manufacturer's instructions (Invitrogen Life Technologies).

Western blot analysis. Total protein from the cells was extracted using RIPA lysis buffer containing $60 \mu \mathrm{g} / \mathrm{ml}$ PMSF. Cell lysates were then subjected to sodium dodecyl sulfate polyacrylamide gel electrophoresis followed by western blot analysis as described previously (28). Protein expression levels in cells tranfected by recombinant plasmid were compared with those transfected by negative plasmid.

Real-time polymerase chain reaction (RT-PCR). RT-PCR was used to detect FASN mRNA levels. Total RNA was extracted from cells using TRIzol reagent (Invitrogen Life Technologies). Total RNA concentration was determined by spectrophotometry at $260 \mathrm{~nm}$ and the purity was determined by calculating the 260/280 ratio with a BioPhotometer (Eppendorf, Hamburg, Germany). RT-PCR and the Two-Step kit (Promega Corporation, Madison, WI, USA) were used to to obtain cDNA according to the manufacturer's instructions, which was then used as the template for amplification. The following primers were used to amplify target sequences: FASN forward 5'-CCCACCTACGTACTGGCCTA-3' and reverse 5'-CTTGGCCTTGGGTGTGTACT-3', 294 bp; $\beta$-actin forward 5'-CGGGAAATCGTGCGTGAC-3' and reverse 5'-TGGAAGGTGGACAGCGAGG-3', 443 bp (Sangon, Shanghai, China). Following amplification, DNA electrophoresis was performed on standard $1 \%$ agarose gels and DNA was labeled and visualised using ethidium bromide. Images were captured using the Canon Digital IXUS 900Ti. FASN mRNA expression levels in cells transfected with recombinant plasmid was compared with cells containing the negative plasmid.

Wound healing assay. Cell migration was assessed by determining the ability of the cells to move into a cellular space in a two-dimensional in vitro 'wound healing assay'. In brief, cells were grown to confluence in 6-well tissue culture plastic dishes to a density of $\sim 5 \times 10^{6}$ cells/well. Cells were denuded by dragging a rubber policeman (Fisher Scientific, Hampton, $\mathrm{NH}$, USA) through the center of the plate. Cultures were rinsed with PBS and replaced with fresh DMEM alone or containing $10 \% \mathrm{FBS}$, following which the cells were incubated at $37^{\circ} \mathrm{C}$ for $24 \mathrm{~h}$. Images were captured at 0 and $24 \mathrm{~h}$ and the migrated distance was measured using ImageJ $(\mathrm{NIH}$, Bethesda, MD, USA). Cell migration rate was calculated using 3 fields/area and presented as the average of 6 independent experiments performed over multiple days. The migration rate of cells transfected by recombinant plasmid targeting the FASN gene, was compared with cells transfected by negative plasmid.

Transwell invasion assay. Invasion of U2-OS cells was measured using the BD BioCoat ${ }^{\mathrm{TM}}$ BD Matrigel $^{\mathrm{TM}}$ Invasion Chamber (BD Biosciences, Franklin Lakes, NJ, USA) according to the manufacturer's instructions. Medium in the lower chamber contained 5\% fetal calf serum as a source of chemoattractants. Cells were suspended in DMEM and added to upper chambers at the same time. Cells that passed through the Matrigel-coated membrane were stained with Diff-Quik (Sysmex, Kobe, Japan) and images were captured under a microscope (ECLIPSE-TS-100, Nikon, Japan; magnification, $\mathrm{x} 400)$ at 0 and $24 \mathrm{~h}$. Cell counts were performed using ImageJ. Values forinvasion wereobtained bycounting 3 fields/membrane and presented as the average of 6 independent experiments performed over multiple days. The number of invaded cells transfected with recombinant plasmid targeting the FASN gene, was compared with cells transfected with negative plasmid.

Statistical analysis. All experiments were repeated 6 times. Data are expressed as the mean \pm SD of $\geq 3$ experiments. Independentsamples T-test was performed for statistical analysis. $\mathrm{P}<0.05$ was considered to indicate a statistically significant difference. All analyses were performed using SPSS version 13.0 (Statistical Software for Social Sciences, Chicago, IL, USA). 


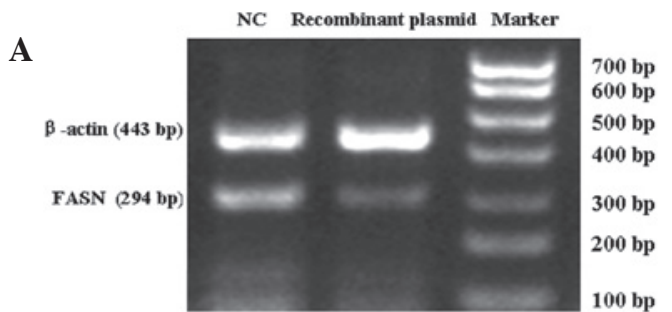

B

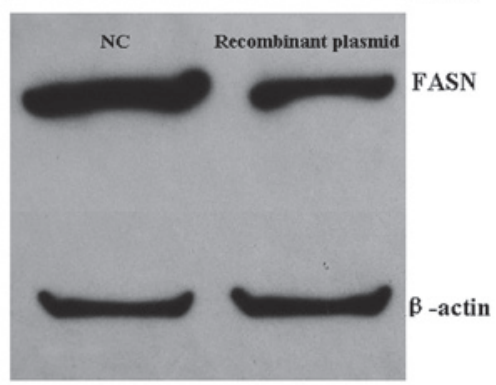

Figure 1. Inhibitory effect of recombinant plasmid on FASN expression. (A) RT-PCR analysis of FASN protein expression in U2-OS cells. FASN mRNA expression was lower in cells transfected with recombinant plasmid compared with negative control. (B) Western blot analysis of FASN protein expression in U2-OS cells. FASN protein expression was reduced in cells transfected with recombinant plasmid compared with negative control. FASN, fatty acid synthase; RT-PCR, real-time polymerase chain reaction; $\mathrm{NC}$, negative control.

A

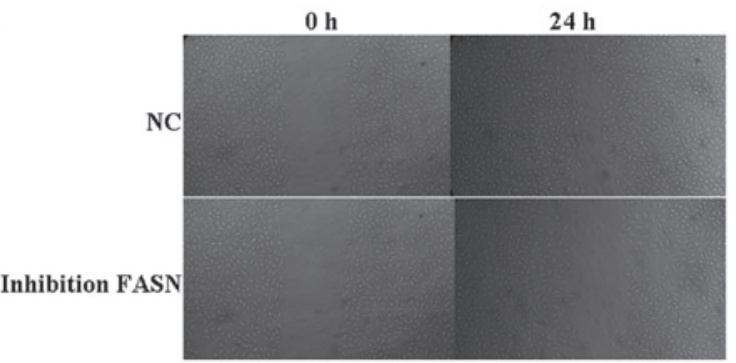

B

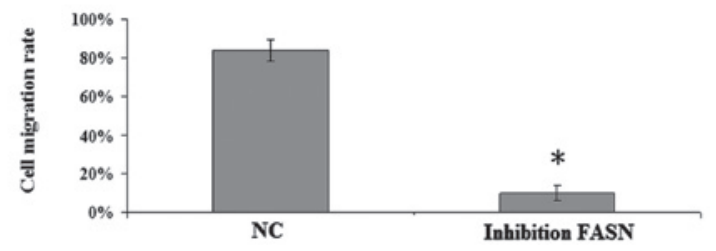

Figure 2. Effect of FASN inhibition by recombinant plasmid on U2-OS cell migration in vitro. (A) Wound healing assay. Migration distance was reduced in cells transfected with recombinant plasmid compared with negative control. (B) Quantification of wound healing assay. Data are presented as the mean $\pm \mathrm{SD}(\mathrm{n}=6) .{ }^{*} \mathrm{P}<0.05$, vs. negative control group. FASN, fatty acid synthase; NC, negative control.

\section{Results}

Effect of recombinant plasmid targeting FASN gene on FASN expression in U2-OS cells. Cultured U2-OS cells were transfected with the recombinant plasmid for $24 \mathrm{~h}$. FASN mRNA and protein expression levels in U2-OS cells were detected by RT-PCR and western blot analysis (Fig. 1). FASN mRNA and protein expression levels in cells transfected with the recombinant plasmid were significantly lower than in those transfected with negative plasmid. These results indicate that recombinant
A

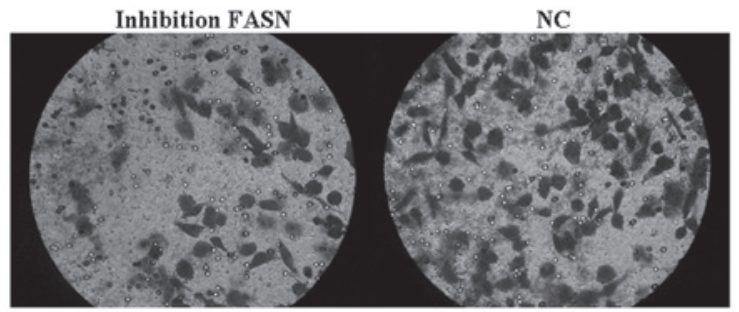

B

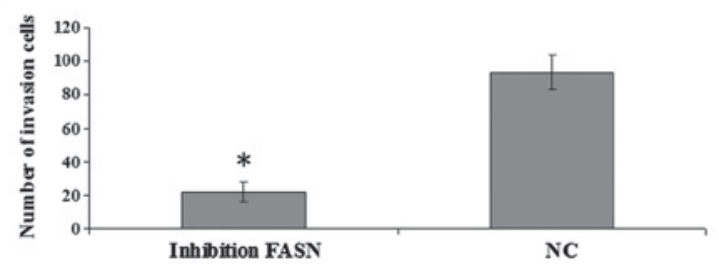

Figure 3. Effect of FASN inhibition by recombinant plasmid on U2-OS cell invasion in vitro. (A) Representative images of Transwell invasion assays using U2-OS cells at $24 \mathrm{~h}$ are presented for each group. (B) Quantification of Transwell invasion assay. The number of invaded cells was significantly lower in cells transfected with recombinant plasmids compared with the negative control. Data are presented as the mean $\pm \mathrm{SD}(\mathrm{n}=6)$. ${ }^{\text {"P }} \mathrm{P}<0.05$, vs. negative control group. FASN, fatty acid synthase; NC, negtaive control.

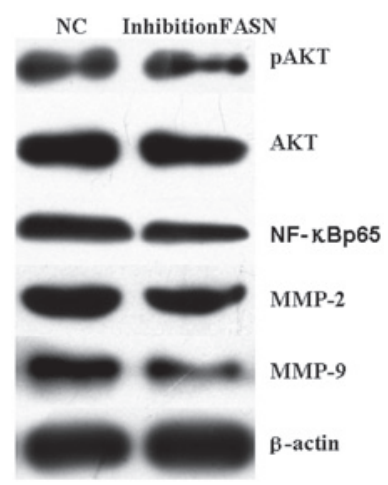

Figure 4. SDS-PAGE analysis of FASN inhibition and Akt phosphorylation and MMP-2, MMP-9 and NF- $\mathrm{KB}$ (p65) protein expression in U2-OS cells. Cells were transfected with recombinant or negative plasmid for $24 \mathrm{~h}$ and protein expression levels of pAkt, MMP-2 and -9 and NF- $\mathrm{kB}$ (p65) were determined. Results indicate that FASN inhibition downregulates phosphorylation of Akt and translocation of NF- $\mathrm{KB}$ (p65) and inhibits expression of MMP-2 and -9 protein. SDS-PAGE, sodium dodecyl sulfate polyacrylamide gel electrophoresis; FASN, fatty acid synthase; MMP, matrix metallopro-

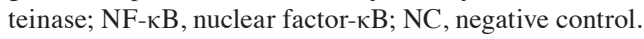

plasmid miRNA targeting the FASN gene may inhibit FASN expression in U2-OS cells.

Effect of FASN inhibition on U2-OS cell invasion and migration in vitro. The recombinant plasmid was transfected into U2-OS cells. Wound healing and Transwell invasion assays were performed to measure the migration and invasion of $\mathrm{U} 2-\mathrm{OS}$ cells and revealed that the number of transmembrane cells $(22.2 \pm 5.6$ cells/membrane) and migration rate $(10 \pm 4 \%)$ in cells transfected by the recombinant plasmid were identified to be significantly reduced compared with cells transfected with negative plasmid (invasion, $93.7 \pm 10.3$ cells/membrane; migration; $84 \pm 5.4 \%$; $\mathrm{P}<0.05$; Figs. 2 and 3 ). The results indicate that FASN inhibition may suppress U2-OS cell invasion and migration in vitro. 
Effect of FASN inhibition on the PI3K/Akt/NF- $\kappa B$ signaling pathway. To investigate the effect of inhibition of FASN on phosphorylation of Akt, expression levels of pAkt protein in U2-OS cells were measured using western blot analysis. Results indicate that pAkt protein expression levels in cells transfected with recombinant plasmid were significantly lower than cells with negative plasmid (Fig. 4). This observation indicated that inhibition of FASN may decrease phosphorylation of Akt. In addition, protein expression levels of NF- $\kappa \mathrm{B}(\mathrm{p} 65)$, MMP-2 and -9 were detected. NF-кB (p65), MMP-2 and -9 protein were decreased significantly in cells transfected with the recombinant plasmid compared with cells transfected with the negative plasmid. These observations demonstrate that inhibition of the FASN gene inhibits MMP-2 and -9 protein expression and nuclear transfer of $\mathrm{NF}-\kappa \mathrm{B}$ in U2-OS cells (Fig. 4) and indicate that silencing FASN may downregulate

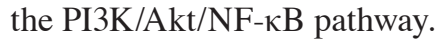

\section{Discussion}

OS is the most common childhood and adolescent primary malignant tumor. Metastasis to the lungs is one of the main causes of mortality in patients with OS. Therefore, study of the molecular mechanisms of metastasis of OS is important to improve survival of patients with metastatic disease.

Human FASN is a $270-\mathrm{kDa}$ cytosolic dimeric enzyme, responsible for fatty acid synthesis. Endogenous fatty acid synthesis from the small carbon precursors acetyl-CoA and malonyl-CoA is dependent on the activity of FASN. In the majority of the cells, FASN is downregulated by dietary fatty acids, with the exception of lipogenic tissues, including the liver, lactating breast, fetal lung and adipose tissue. Previous studies have identified that neoplastic lipogenesis is essential for cancer cell survival (29). In addition, downregulation of FASN has been revealed to decrease invasion and migration in a variety of human tumors $(19,20)$. Previously, we reported that cerulenin, an inhibitor of FASN inhibits OS cell proliferation in vivo and in vitro (30). However, it is currently unknown whether silencing FASN suppresses OS cell invasion and migration and the molecular mechanisms associated with this process have yet to be defined. In the present study, a recombinant plasmid, containing miRNA designed to target the FASN gene, was constructed for inhibition of FASN in OS cells. The inhibitory effect was investigated by RT-PCR and western blot analysis and the results demonstrated that FASN expression levels were significantly inhibited by the recombinant plasmid (Fig. 1). In addition, wound healing and Transwell invasion assays were performed to detect the migration and invasion of U2-OS cells. The migration rate of cells transfected by the recombinant plasmid was identified to be significantly lower than the negative plasmid cells (Fig. 2). Invasion was also inhibited (Fig. 3). Results indicate that silencing the FASN gene may inhibit OS cell invasion and migration in vitro.

The molecular mechanisms associated with FASN silencing and inhibition of OS cell migration and invasion were also analyzed. The role of the PI3K/Akt/NF- $\kappa \mathrm{B}$ signaling pathway in OS invasion and migration was confirmed. Upregulation of FASN expression in cancer cells has been previously associated with the PI3K/Akt signaling pathway $(31,32)$. In addition, FASN inhibition leads to downregulation of activated Akt and its downstream targets (33-35). Akt is essential for $\mathrm{NF}-\kappa \mathrm{B}$ activation by stimulation of the IKK complex, which phosphorylates and inactivates $\mathrm{I} \kappa \mathrm{B}$, an inhibitor of $\mathrm{NF}-\kappa \mathrm{B}$. NF- $\kappa \mathrm{B}$ is composed of DNA-binding subunits (p50 and p52) and subunits with transcriptional activity (p65 and RelB or c-Rel), which dimerize in various combinations. The primary form of $\mathrm{NF}-\kappa \mathrm{B}$ is a heterodimer of the p50 and p65 subunits and is localized mainly to the cytoplasm in an inactive form bound to I $\mathrm{B}$. Previously, NF- $\kappa \mathrm{B}$ was demonstrated to upregulate MMP-9 (36). In addition, inhibition of $N F-\kappa B$ was identified to downregulate MMP-2 (37). During the development of metastases, cancer cells must degrade the components of the extracellular matrix. MMPs, particularly MMP-2 and -9, are markedly associated with this process due to their capacity to degrade the extracellular matrix, promoting tumor invasion.

In the present study, pAkt protein expression levels were detected by western blot analysis to investigate whether silencing FASN led to downregulation of the PI3K/Akt/NF- $\kappa \mathrm{B}$ signaling pathway. Expression of pAkt protein was decreased in FASN-inhibited compared with negative control cells (Fig. 4), indicating that FASN inhibition downregulates phosphorylation of Akt. In addition, western blot analysis was performed to investigate expression levels of NF- $\kappa \mathrm{B}$ (p65) and MMP-2 and -9 protein. Again, protein expression levels were reduced in FASN-inhibited compared with negative control cells (Fig. 4), indicating that FASN inhibition reduces nuclear translocation of NF- $\kappa \mathrm{B}$ and attenuates activation of MMP-2 and -9 protein.

The present study demonstrates that inhibition of FASN may suppress OS cell invasion and migration via downregulation of the PI3K/Akt/NF- $\kappa \mathrm{B}$ pathway in vitro. Results indicate that targeting FASN and the PI3K/Akt/NF- $\kappa \mathrm{B}$ pathway may be a potential treatment strategy for treating OS metastases.

\section{Acknowledgements}

The present study was supported by grants from the National Natural Science Foundation of China (No. 81260400) and the Natural Science Fundation of Jiangxi Province (No. 20114BAB205093).

\section{References}

1. Meyers PA, Schwartz CL, Krailo M, et al: Osteosarcoma: a randomized, prospective trial of the addition of ifosfamide and/ or muramyl tripeptide to cisplatin, doxorubicin, and high-dose methotrexate. J Clin Oncol 23: 2004-2011, 2005.

2. Bacci G, Forni C, Longhi A, et al: Local recurrence and local control of non-metastatic osteosarcoma of the extremities: a 27-year experience in a single institution. J Surg Oncol 96: 118-123, 2007.

3. Jawad MU, Cheung MC, Clarke J, et al: Osteosarcoma: improvement in survival limited to high-grade patients only. J Cancer Res Clin Oncol 137: 597-607, 2011.

4. Mialou V, Philip T, Kalifa C, et al: Metastatic osteosarcoma at diagnosis: prognostic factors and long-term outcome - the French pediatric experience. Cancer 104: 1100-1109, 2005.

5. Hegyi M, Semsei AF, Jakab Z, et al: Good prognosis of localized osteosarcoma in young patients treated with limb-salvage surgery and chemotherapy. Pediatr Blood Cancer 57: 415-422, 2011.

6. Stokkel MP, Linthorst MF, Borm JJ, et al: A reassessment of bone scintigraphy and commonly tested pretreatment biochemical parameters in newly diagnosed osteosarcoma. J Cancer Res Clin Oncol 128: 393-399, 2002. 
7. Yeh CS, Wang JY, Cheng TL, et al: Fatty acid metabolism pathway play an important role in carcinogenesis of human colorectal cancers by microarray-bioinformatics analysis. Cancer Lett 233: 297-308, 2006.

8. Hess D and Igal RA: Genistein downregulates de novo lipid synthesis and impairs cell proliferation in human lung cancer cells. Exp Biol Med (Maywood) 236: 707-713, 2011.

9. Alo PL, Amini M, Piro F, et al: Immunohistochemical expression and prognostic significance of fatty acid synthase in pancreatic carcinoma. Anticancer Res 27: 2523-2527, 2007.

10. Walter K, Hong SM, Nyhan S, et al: Serum fatty acid synthase as a marker of pancreatic neoplasia. Cancer Epidemiol Biomarkers Prev 18: 2380-2385, 2009.

11. Okawa Y, Hideshima T, Ikeda H, et al: Fatty acid synthase is a novel therapeutic target in multiple myeloma. Br J Haematol 141: 659-671, 2008

12. Migita T, Ruiz S, Fornari A, et al: Fatty acid synthase: a metabolic enzyme and candidate oncogene in prostate cancer. J Natl Cancer Inst 101: 519-532, 2009

13. Silva SD, Cunha IW, Younes RN, et al: ErbB receptors and fatty acid synthase expression in aggressive head and neck squamous cell carcinomas. Oral Dis 16: 774-780, 2010.

14. Orita H, Coulter J, Tully E, et al: Inhibiting fatty acid synthase for chemoprevention of chemically induced lung tumors. Clin Cancer Res 14: 2458-2464, 2008.

15. Saati GE and Archer MC: Inhibition of fatty acid synthase and Sp1 expression by 3,3'-diindolylmethane in human breast cancer cells. Nutr Cancer 63: 790-794, 2011.

16. Notarnicola M, Pisanti S, Tutino V, et al: Effects of olive oil polyphenols on fatty acid synthase gene expression and activity in human colorectal cancer cells. Genes Nutr 6: 63-69, 2011.

17. Notarnicola M, Messa C, Refolo MG, et al: Polyunsaturated fatty acids reduce fatty acid synthase and hydroxy-methyl-glutaryl CoA-reductase gene expression and promote apoptosis in HepG2 cell line. Lipids Health Dis 10: 10, 2011.

18. Zecchin KG, Rossato FA, Raposo HF, et al: Inhibition of fatty acid synthase in melanoma cells activates the intrinsic pathway of apoptosis. Lab Invest 91: 232-240, 2011.

19. Carvalho MA, Zecchin KG, Seguin F, et al: Fatty acid synthase inhibition with Orlistat promotes apoptosis and reduces cell growth and lymph node metastasis in a mouse melanoma model Int J Cancer 123: 2557-2565, 2008.

20. Murata S, Yanagisawa K, Fukunaga K, et al: Fatty acid synthase inhibitor cerulenin suppresses liver metastasis of colon cancer in mice. Cancer Sci 101: 1861-1865, 2010.

21. Zhang XX, Fu Z, Zhang Z, et al: Microcystin-LR promotes melanoma cell invasion and enhances matrix metalloproteinase-2/-9 expression mediated by nf-kappab activation. Environ Sci Technol 46: 11319-11326, 2012.

22. Kao SJ, Su JL, Chen CK, et al: Osthole inhibits the invasive ability of human lung adenocarcinoma cells via suppression of NF-kappaB-mediated matrix metalloproteinase-9 expression. Toxicol Appl Pharmacol 261: 105-115, 2012.
23. Kuan YH, Huang FM, Li YC, et al: Proinflammatory activation of macrophages by bisphenol A-glycidyl-methacrylate involved NFkappaB activation via PI3K/Akt pathway. Food Chem Toxicol 50: 4003-4009, 2012.

24. Swinnen JV, Van Veldhoven PP, Timmermans L, et al: Fatty acid synthase drives the synthesis of phospholipids partitioning into detergent-resistant membrane microdomains. Biochem Biophys Res Commun 302: 898-903, 2003

25. Uddin S, Siraj AK, Al-Rasheed M, et al: Fatty acid synthase and AKT pathway signaling in a subset of papillary thyroid cancers. J Clin Endocrinol Metab 93: 4088-4097, 2008.

26. Wang HQ, Altomare DA, Skele KL, et al: Positive feedback regulation between AKT activation and fatty acid synthase expression in ovarian carcinoma cells. Oncogene 24: 3574-3582, 2005.

27. Menendez JA, Vellon L, Mehmi I, et al: Inhibition of fatty acid synthase (FAS) suppresses HER2/neu (erbB-2) oncogene overexpression in cancer cells. Proc Natl Acad Sci USA 101: 10715-10720, 2004.

28. Bai X, Ma D, Liu A, et al: Rheb activates mTOR by antagonizing its endogenous inhibitor, FKBP38. Science 318: 977-980, 2007.

29. Li W, Tai Y, Zhou J, et al: Repression of endometrial tumor growth by targeting SREBP1 and lipogenesis. Cell Cycle 11: 2348-2358, 2012.

30. Liu ZL, Wang G, Shu Y, et al: Enhanced antitumor activity of epirubicin combined with cerulenin in osteosarcoma. Mol Med Report 5: 326-330, 2012.

31. Bandyopadhyay S, Pai SK, Watabe M, et al: FAS expression inversely correlates with PTEN level in prostate cancer and a PI 3-kinase inhibitor synergizes with FAS siRNA to induce apoptosis. Oncogene 24: 5389-5395, 2005.

32. Porstmann T, Griffiths B, Chung YL, et al: PKB/Akt induces transcription of enzymes involved in cholesterol and fatty acid biosynthesis via activation of SREBP. Oncogene 24: 6465-6481, 2005.

33. Tomek K, Wagner R, Varga F, et al: Blockade of fatty acid synthase induces ubiquitination and degradation of phosphoinositide3-kinase signaling proteins in ovarian cancer. Mol Cancer Res 9: 1767-1779, 2011

34. Zaytseva YY, Rychahou PG, Gulhati P, et al: Inhibition of fatty acid synthase attenuates CD44-associated signaling and reduces metastasis in colorectal cancer. Cancer Res 72: 1504-1517, 2012.

35. Uddin S, Jehan Z, Ahmed M, et al: Overexpression of fatty acid synthase in Middle Eastern epithelial ovarian carcinoma activates AKT and Its inhibition potentiates cisplatin-induced apoptosis. Mol Med 17: 635-645, 2011.

36. Andela VB, Gordon AH, Zotalis G, et al: NFkappaB: a pivotal transcription factor in prostate cancer metastasis to bone. Clin Orthop Relat Res (415 Suppl): S75-85, 2003.

37. Felx M, Guyot MC, Isler M, et al: Endothelin-1 (ET-1) promotes MMP-2 and MMP-9 induction involving the transcription factor NF-kappaB in human osteosarcoma. Clin Sci (Lond) 110: 645-654, 2006. 